\title{
Posthumous personhood and the affordances of digital media
}

Today a number of digital applications and services can offer individuals some sort of posthumous social presence and agency. Some start up companies promise to keep Twitter accounts active after the death of the account holder, and others will send a series of emails to selected parties after an individual's death. As other researchers have already noted, social media platforms have also been implicated in the continuing social presence and agency of the dead (Karppi, 2013; Marwick and Ellison, 2012). More dramatically, a few businesses are attempting to build interactive online avatars, so family and friends can continue to interact with loved ones after their death. These developments raise significant temporal and ontological issues for personhood within and beyond digital environments (Graham et al., 2013; Stokes, 2012). In the following paper, we identify the different ways that digital media can extend one's personhood. We suggest that these forms of posthumous personhood, through the unique affordances of digital media, challenge and reshape our existing understandings of the boundaries drawn between life and death.

The paper begins by discussing understandings of personhood and posthumous social agency, through both the symbolic and material continuities between the living and the dead identified within anthropological and sociological literature (see Hallam and Hockey, 2001; Lifton, 1979). Placing this literature in conversation with work in Science and Technology Studies (Arnold, 2002; Latour, 2004, 2005) we go on to suggest that digital codes and computational texts are key sites for posthumous personhood. Through a brief descriptive outline drawn from commercial and popular examples, we then show how various digital resources are being used to create different forms of posthumous personhood. Finally, in our discussion we situate these forms of digital posthumous personhood in relation to a wider discursive politics of social media (Lambert, 2013; Light and Cassidy 2014; Light, 2014), which we suggest directly supports the maintenance of ongoing interactions with the dead online.

\section{Theories of posthumous personhood}

Our examination of posthumous personhood begins with a brief definitional discussion. The etymology of personhood derives from the Latin persona and it is from this concept of a "fundamental entity" that the contemporary category of "a person" developed (Frow, 2012). The Roman conceptualisation of persona is notable because it is clear that from the beginning of these etymological origins, personhood did not refer to a singular, biological human being, delimited by the body. Roman law allowed a biological individual to adopt any one of multiple personas depending upon social and legal context. Furthermore, under law some biological humans, such as slaves, were not viewed as persons. Roman citizens were a subset of biological humans who were by default persons, but lost personhood if taken into slavery by an enemy of Rome. They regained it automatically and retrospectively upon return to Rome. However, if they were still classified as slaves at their time of biological death, they were regarded as being dead not from the time of biological death but from the moment of enslavement (Frow, 2012). 
Subjects and bodies have thus shown potential disassociation over millennia. Of course, a number of more contemporary definitions of personhood imply that a subject should be alive. For example Daniel Dennett's (1988) definition requires that persons be 'rational beings' (p. 177), be 'capable of verbal communication' (p. 178) and be 'conscious' (p. 178) amongst other things. Another definition provided by John Tehranian (2011, p. 59), suggests that a person needs to be able to interact and communicate in order to 'form' and 'express' one's personhood:

Formation of personhood takes place internally as an individual's identity is shaped through interaction with objects in the external world. Meanwhile, the expression of personhood occurs when the individual communicates some aspect of her (already formed) identity to others as a way of contextualizing herself, through her relationship with objects, within the broader community.

However, both definitions leave a space open for the deceased to retain a semblance of personhood by locating personhood within a 'relational' position (Latour 2005) of interaction, not as an ontological absolute. Karl Smith's (2012, p. 61) review of the long debate in anthropology around the distinction between the 'dividual' and 'individual' reminds us that 'every human child enters the world open to the socialising forces of its cultural milieu', in a 'porous' state vis-à-vis the influence of others. It would therefore follow, that every human being leaves the world also in such a 'porous' relational state of personhood.

This notion has been explored by a range of scholars, who have suggest that the deceased retain some sort of posthumous personhood. For example, Robert J. Lifton (1979, see also see Vigilent and Williamson 2003) outlines five ways that people have sought to achieve a form of 'symbolic immortality': Biological immortality (through biological reproduction and social attachments); creative immortality (creative production); transcendental immortality (overcoming death through religion e.g. eschatology); natural immortality (recognising the world will remain); and experiential transcendence, which involves entering a psychic state where notions of time and death disappear. These forms of immortality all carry significant implications for identity and personhood as it means that a person's identity does not cease to exist after their 'corporeal demise', nor does 'their corporeal demise ... preclude postmortal interactions with loved ones left behind' (Vigilant and Williamson, 2003, p. 180).

Anthropological research has also revealed 'that, for many cultures, the social, symbolic and mnemonic significance of the dead body does not end with the extinguishing of vital signs' (Williams, 2004, p. 265). Robert Hertz (2004 [1907]) drew on a great range of ethnographic material from around the world at the turn of the $20^{\text {th }}$ century to show that death is a process rather than an event. He described 'double funerals' observed in Indonesia and Madagascar, which not only separated the individual 'person' from the collective but re-established order in society through the reallocation of duties. These procedures continue in contemporary Western societies with death never seen as a 'straightforward moment or event' but rather 'an outcome of the social relations through which the categorisation of death is negotiated' (Hallam et al. 1999, p. 57). 
Various scholars have echoed this position by developing the initial theories of Latour (2004, 2005) and Gell (1998), both of whom view non-human objects as having agency (Hallam and Hockey 2001; Hockey et al. 2010; Williams 2004). This has seen scholars reposition the corpse as a still active agent and indeed the central 'focus for personhood and remembrance of the dead in mortuary practices' (Williams, 2004). However, as Kevin O'Neill notes, there are limits to what the corpse can do, and indeed, what it can be. On the one hand, an embalmed corpse 'allows those left behind to re-call ... the soul that is departed, and remember' but the practice of embalming cannot 'connect the living with that still-living essence' (O'Neill, 2008, p. 179). On the other hand, the increased use of interactive media on both online and physical memorials, allows the dead to live 'a life that looks more coherent and consequential than that left in individual memories' (O’Neill 2008, p. 182).

We explore this conundrum by examining how these recent attempts at maintaining some sort of online social agency and identity after death alter our existing understandings of posthumous personhood. We take the notion of 'distributed personhood' as a point of departure for our article, a concept that recognises 'a model of the individual which transcends the boundaries of the body' (Hockey et al. 2010: 9). In the following section, we will outline a series of recent practices and services, which allow one's 'being (agency, subjectivity and identity)' to move beyond the corporeal body and the boundaries of the skin (Arnold, 2002, p. 234). We suggest that if the dead can now tweet or indeed be re-animated through an avatar, the question of the dead's status as person now turns not just on their biological status, nor on their status as active social participants, but also on their technologically-mediated status and interactive presence in the world (Stokes, 2012).

\section{Establishing a posthumous presence online}

The maintenance of a posthumous social media presence can be delegated to a growing number of companies, who have recognised a market opportunity in providing a managed response to death (see Kearl, 2010). Death Switch for example, sends a client a regular email prompting him or her to enter a password 'to make sure you're still alive' (http://deathswitch.com/). Failure to respond to the email automatically activates a prescripted message from the client's email account to nominated recipients, notifying them of the client's death. This allows the client to deploy a pre-planned strategy of posthumous digital asset management, involving basic password storage and forwarding. However, one could also automate the destruction of 'unspeakable secrets' or issue messages to loved ones, or score the 'last word in an argument' (http://deathswitch.com/).

These communicative actions can occur through a range of mediums and platforms, and software code allows for posthumous digital messaging to be automated and ongoing. Delivery mechanisms range from email (as seen above) and blog posts, to text and tweets. If I Die (http://ifidie.net/) for example, allows a client to create a video or text message that will only be posted to Facebook posthumously. A nominated trustee will let the service know that the client is no longer alive, and then the video or message will be automatically distributed through the client's social media accounts. In a slightly more complex fashion, DeadSoci.al (http://www.deadsoci.al/) allows users to 'upload an unlimited number of photos, video, 
audio and text messages which will be sent out on Facebook, Twitter, LinkedIn and the DeadSoci.al website after their death' (Kleenman, 2014). These messages are prepared and uploaded pre-death, and are then scheduled for distribution through a variety of social media platforms up to 999 years into the future. Clients may for example pre-record messages to be sent on the anniversary of their death, on each birthday of their partner or on the event of their grandchild's $21^{\text {st }}$ birthday. All an executor need do in this case, is activate the program, and the pre-recorded videos, emails and tweets will be sent out according to schedule. Alternatively, services like LivesOn rely on algorithms in order to maintain a digital presence. LivesOn utilises software programs to analyse a person's Twitter feed, 'learning' about likes, tastes and syntax, and then algorithmically generates tweets that reflect the user on their behalf. LivesOn attempts to predict what one might tweet next, and uses the slogan 'When your heart stops beating, you'll keep tweeting'. One can delegate responsibility for one's Tweets to LivesOn, allowing it to 'be you' in the world of the Twitter stream after your death.

Services like Deadsoci.al make heroic assumptions regarding their own longevity. They present a largely static view of a future networked world, which assumes that services like Facebook will continue to be relevant and remain in service in the future and, perhaps playing on the hubris of their clients, assumes that personal messages recorded in the current day will be valued into the middle and far future. However, Deadsoci.al CEO James Norris has few doubts about the service's social and emotional relevance, and 'apparently has been taking the emotional aspect of the app seriously', with the company 'consulting with a doctor specialising in end-of-life care' (Coldwell, 2013). Norris identifies two reasons for a consumer using Deadsoci.al, as an 'end-of-life tool' or as a way of extending friendships. He notes that while 'you can't receive messages' the service allows you to 'think about how you want your friendships to continue' (Kleenman, 2014).

Another way to enhance posthumous social connectivity sees see the online persona of an individual maintained after death on social media, through the personal intervention of friends or family. There are various ways to manage the social media profile of someone who has died. On Facebook, the profile of a dead person can simply remain "live", removed entirely or placed in a memorialised form so only those already 'friended' can access the page (Odom et al., 2010). On most other social media platforms, there are only two choices available: delete the profile or keep it active. When a profile stays online, it can become a memorial site for the bereaved, where the living can recount their relationship with the dead over an extended period of time (see Brubaker and Hayes, 2011). However, in other cases ongoing posts are made on behalf of the dead person by the living.

One of the more notable examples of this practice is the continued presence of popular film critic Roger Ebert on Twitter. A prolific film critic and writer, Roger Ebert suffered from neck and face cancer late in life, which left him unable to talk (Jones, 2010). However, he was still able to write and he subsequently built a large following on Twitter (Ebert, 2010). But his tweets did not stop after his death in April 2013. Instead his wife Chaz Ebert continues to operate the account on her husband's behalf, posting links to old reviews and 
photos and using the account to publicise a documentary about her late husband. She also occasionally presents her own voice, prefacing her own tweets with 'Chaz'.

The act of controlling the account of a deceased person is not just limited to celebrities. In Auckland, for example, a case was published concerning Greg Murphy, the husband of deceased cancer patient Natalie, who maintained his wife's Facebook profile after her death and updated it regularly (Russell, 2014). To Natalie's Facebook friends, it appeared that she was still posting comments. Greg used her profile to continue what he saw as 'her' advocacy work, raising awareness for breast cancer prevention and networking for related fundraising events. He notes that some 'friends were shocked when Natalie appeared in their newsfeed but most have greeted it with positivity... [though] the process is not without some strange encounters'. He ends by acknowledging that there is a 'darker side', and recalls that some men have attempted to "hit on Natalie" unaware that while her profile is active, she is actually dead (Russell, 2014).

Insomuch as posting to social media can be described as living, these examples clearly allow the digital persona to maintain some sort of a social life after death. Roger Ebert for example appears to have retained some sort of professional immortality, with his critical opinion, industry connections and fan base lasting well beyond his death. The fact that Natalie was also "hit on" after death gestures to the 'flatness' of the screen and the blurred lines between 'representation' and personhood proper. The very fact of a social media profile implies a live person on the other end and if surrogates posting do not make their status clear, it is difficult for strangers and acquaintances to know that this person, who may be social and engaging online, is actually dead. This means that either businesses or family and friends can often establish a very close approximation of the deceased's persona, when either using or posting to the deceased's social media profile.

\section{Potential futures of Posthumous Personhood}

While these examples might be technically primitive, such forms of posthumous connection are gradually being superseded by a range of research and development projects which imagine a kind of digitally mediated immortality (see Lombard and Selverian, 2008). For example, in the 2011 TED talk, 'After Your Final Status Update', new media commentator Adam Ostrow argues that the basic tools available today are just the tip of the iceberg. Computers will soon be able to:

[U]nderstand human language and process vast amounts of data [...] It's going to become possible to analyse an entire life's worth of content - the tweets, the photos, the videos, the blog posts that we are producing in such massive numbers. And I think as that happens it's going to become possible for our digital personas to continue to interact in the real world long after we're gone thanks to the vastness of the amount of content we're creating and to technology's ability to make sense of it all (Ostrow, 2011).

Ostrow goes on to anticipate a future where the collective content uploaded will be embedded in robotic or holographic representations of the deceased, which could interact with the living based on the archive of content produced by a person over a lifetime. He thinks this is possible once the amount of data we are producing and technology's ability to understand it both expand exponentially: 'Why not upload the collective of your online interactions into a 
robot, or project your personality as a hologram to go on interacting with your family and friends after you die?'

One of the first examples to use digital technologies to reconstruct a version of life after death was Virtual Eternity (www.virtualeternity.com via archive.org; see Stokes, 2012). Developed by Intellitar Inc. virtual eternity was one application among their so-called 'intelligent avatar platform' (IAP) which allowed people to create digital 'clones' or 'intellitars', a portmanteau of 'intelligent' and 'avatar', for use in a number of commercial and personal contexts. Specifically, the 'live' beta release of Virtual Eternity promised to allow people to begin creating their unique digital legacy:

Intellitar ${ }^{\mathrm{TM}}$ is the developer of the Intelligent Avatar Platform ${ }^{\mathrm{TM}}$ (IAP) and creator of Virtual Eternity ${ }^{\mathrm{TM}}$. Founded in 2008, Intellitar is delivering the first intelligent and interactive technology platform for building and creating life-like avatars or an "Intellitar". The IAP allows a user to create and train his or her Intellitar to accurately reflect the personality, voice, look, knowledge and life experiences of its creator...

Intellitar's Virtual Eternity brings the power of the Intelligent Avatar Platform to genealogy by allowing users to build and enhance their individual or family legacy. Now, with Virtual Eternity, generations can be digitally preserved to allow future interaction and conversation with friends and family members (www.virtualeternity.com via archive.org).

Intellitar Inc. envisioned leveraging the IAP platform to allow avatars to operate autonomously across other social network sites. A free account was made available but the options available were limited, for example, by only allowing the use of off-the-shelf prepared digital voices. In contrast, paid accounts included a personalised voice achieved by recording a series of words and phrases. In addition, 'intellitars' featured a digital photo portrait uploaded as a .jpeg image that could move using digital animation, and included a 'personality' based on a script of questions covering personal information and memories, providing the data for an artificial intelligence engine to extract answers to questions and engage in conversation.

In our engagements with the service, the limitations of the technology were readily apparent. The digital animation was far from life-like, with lip and facial movements appearing like a clumsy identity-kit, and the lack of intelligence was clear the minute one asked a question outside the script. The fact that the service is now defunct underlines the large divide between ambition and practicality in this space. However, the service did reveal a semblance of interaction. It could by no means pass the Turing test and was not particularly adaptive to communicative context, but it did operate as an archive of a deceased life that could be interrogated by the living.

Eterni.me represents a more recent instantiation of this move towards the creation of an adaptive digital presence. This company is extending artificial intelligence techniques in order to build a virtual copy of a person. The individual is expected to train their avatar prior to death 'through daily interactions, in order to improve its vocabulary and conversational skills' (Parker, 2014). This representation of an individual's personality is enhanced through algorithmic methods, pattern matching and data mining. The company will collect 'almost 
everything that you create during your lifetime and process ... this huge amount of information using complex Artificial Intelligence algorithms' (http://eterni.me/). This will incorporate data from 'Facebook, Twitter, e-mail, photos, video, location information, and even Google Glass and Fitbit devices' (Parker, 2014). A public launch could be up to five years away, as the company waits for artificial intelligence research to catch up and be able to execute increasingly sophisticated algorithms on personal data.

\section{Posthumous Personhood and the Politics of Digital Culture}

This outline of the current digital environment reveals an expanding range of ambitions to extend posthumous existence, creating new opportunities for personas of the deceased to communicate and interact in the world of the living. This may be through the assistance of a loved one assuming the dead person's profile, or through the stuttering efforts of algorithms and their software programs. However, as the work of Intellitar and Eterni.me highlight, there is scope in the future for increasingly complex posthumous engagements to occur. Regardless of how these interactions transpire, we argue that all of the above examples materialise new forms of persona that persist beyond death and establish relations with the living.

These interactions are ontological, rather than representative. In each example the body does not directly intercede (whether living or dead) and the 'role' of the person in question is performed entirely through networked, digital data, text, narrative and image (Arnold, 2002). According to the 'pragmatic' school of communications (Coyne, 1998), the text itself acts in the world, and the manner in which it acts is derived from the text's relations to other forms of narrative, and the narrative's relations to emotional affect, memory, the psyche, and so on. This narration of a world is a kind of action that 'makes it so'. In this 'pragmatic' view of textual narrative, what appears on social media is not a simulacra or a metaphor for embodied relations, it is the locus of agency and being in the (online) world. In the digital afterlife, the text or the video on the screen is not a simulacrum for ongoing relations, it is not representative, the text or the video is an ongoing form of relation.

Indeed, in each example above we see an active social relationship established. The tactful appropriation of the social media profile of a deceased person can be seen as a tribute to ongoing sociality. Pre-recorded messages deployed through services like Deadsocial are based around the notion of maintaining ongoing friendships with no need for both friends to be alive. Finally, the after-lives through adaptive digital presence proposed by futurists offer clear capacity for the deceased to maintain some level of active agency in everyday life. For these reasons, as well as in light of the ontological claims made in the paragraphs above, we suggest that these technologies also function as a form of 'distributed personhood' (Nansen et al. 2014; 2015).

As the above suggests, our claims draw on studies conducted around alternative sites of distributed personhood. However, we suggest that the contexts in which these services and practices are being deployed are deserving of further critical analysis. The relative popularity of some of these examples - such as posthumous personhood through forms of digital surrogacy - underlines the fact that today memorialising and remembering is implicated into 
a wider commercial start up economy. Ben Light and Elija Cassidy note that social networks are generally framed through a 'lens of connectivity' (2014, 2), with both commercial and scholarly discourses emphasising connectivity and connection. A complex data economy also assists in entrenching connection as a social practice. As Tero Karppi (2013) points out, it is significantly easier to memorialise a page on Facebook rather than delete it. This is because memorialising an account allows Facebook to keep the dead user's data within their data economy. Finally, Lambert $(2013,80)$ emphasises the extent to which online connection can shape one's very ontological condition:

\section{The negation of a social tie is a negation of self. Hence, the prevention of loss has existential dimensions. Reclaiming ties on Facebook is an existential process. It chooses being over nothingness, presence over absence.}

This discursive terrain reveals some not unexpected motivations behind the commercial and social push for the preservation of posthumous personhood - the prevention of loss and the continuation of 'self - and suggest that these sorts of interactions are going to be supported by commercial interests for some time. ${ }^{1}$

If these sort of posthumous social engagements are likely to continue, the next question then is what sort of relationships will they form? Whereas a corpse or a material object only retains a limited form of agency, as the above examples suggest, there may come a time where the dead are making direct interventions into our social lives. Brubaker and Vertesi (2010) propose thinking about the dead on social networks as examples of 'extreme users', rather than as non-users or former users. We suggest that while the term 'extreme' may capture some elements of their ontological status within social media, the ways in which these relationships are enacted are quite common and not particularly unique nor removed from ordinary online life. In short, we suggest that in our contemporary digital culture, relationships between posthumous and living persons may not be dramatically different from existing online friendship practices.

As Lambert notes, distant intimacy is already a common feature on mainstream social network sites and is 'wrapped up with the prevention of loss' $(2013,80)$. Facebook offers users the chance to connect with lost friends and also sustain distant ties, 'which are objects of occasional interest' $(2013$, p. 87). This description could also fit the relationship between a living person and a dead Facebook friend. After the initial grieving period, the profile pages of the deceased are typically only engaged with during landmark dates, such as birthdays or at the anniversary of a death (Brubaker and Hayes, 2011). In short, like old high school friends or workmates, the dead become distant ties, and one can check in at key moments of their (past) life. In the case of surrogate-supported accounts, posthumous interactions will be driven by a living human (who assumedly knew the deceased well), and so ongoing engagements representing the deceased will be able to be sensitively and authentically managed.

\footnotetext{
${ }^{1}$ These interactions are also supported by start up culture in general, which has a particularly utopian bent (Barbrook and Cameron, 1996), as well as (arguably) the culture of the United States (see Kearl, 2010).
} 
Other examples discussed above may be similarly productive. Services like Deadsoci.al may be unable to support contextual and ongoing practices of intimacy and reciprocity that define a contemporary family or friendship (Lambert, 2013). However, these messages delivered from beyond the grave will retain some agency and be able to call upon a particular form of posthumous personhood. These encoded and textual forms of vernacular posthumous engagement have a long media history and are perhaps best suited to the ongoing relational engagements that assist in the formation of personhood (see Gell, 1998; Tehranian, 2014). In a strange way then, the most advanced forms of technology - algorithmically driven digital presences - may turn out to be the most awkward, at least at this point in time. These services are not intuitive and present a poor and perhaps unsettling performative re-enactment of the individual. These efforts to create posthumous persons can be more jarring than recollecting a person, while reading their posthumous words, or even engaging in a conversation with their corpse (Harper, 2010) and they currently present the most difficulties when attempting to maintain a relationship with the deceased.

\section{Conclusion}

This paper has extended existing notions of de-centred personhood (Hallam et al. 1999; Hallam and Hockey 2001; Hockey et al. 2010; Gell, 1998) by outlining various forms of posthumous social personhood. We have gone on to describe the ambitions of symbolic immortality evident in the deployment of networked media for the purposes of enacting posthumous social personhood. These attempts at digital incarnation materially reconstitute the pre-deceased into the deceased (Harper, 2010) and form part of a historical trajectory of efforts at symbolic immortality (Lifton, 1979). We suggest that the affordances of online platforms now contribute to a technosocial context where the boundary between the living and the dead becomes increasingly indistinguishable, and at particular points even inconsequential. We suggest that if the persona of the dead can still act in the world through the same forms of technical mediation that maintain the social life of the living, they may be considered as a kind of 'person'.

If the deceased do retain some kind of personhood, it then follows that a broader ethics of interaction with the dead needs to emerge. The dead have been communicating with us for a long time (consider when letters or journals are left for family and friends to read after an individual dies), but the persistent, searchable, replicable and scalable (Marwick and Ellison, 2012) nature of digital content presents a series of new problems for us to confront. The banal nature of interacting with a deceased friend on social media, which we outline above, underlines the fact that the digital space is an arena where the worlds of the living and dead are blurred. This in turn raises the issue of how one should speak to the dead. Of course, as we note above, steps have been made towards this. Social media platforms offer a range of solutions for posthumous account management and intimate relations of the deceased are establishing methods of genuine engagement with the posthumous identities through their everyday practice. However, if the predictions of Ostrow are fully realised and avatar services like Eterni.me improve substantially, ethical questions around interacting with the dead will become a pressing social concern. It therefore makes sense to view our existing 
interactions with the deceased on social media platforms as an ethical test ground, a space where a range of approaches to the phenomenon of posthumous personhood can occur, before any further technological advances occur.

\section{References}

Arnold, M. (2002). The glass screen. Information, Communication \& Society, 5(2), 225-236.

Barbrook, R., \& Cameron, A. (1996). The Californian Ideology. Science as Culture, 6(1), 4472.

Brubaker, J. \& Hayes, G. (2011). "We will never forget you [online]": An empirical investigation of post- mortem MySpace comments. Proceedings of the 14th Conference on Computer Supported Cooperative Work, 123-132.

Brubaker, J. R., \& Vertesi, J. (April, 2010). Death and the social network. Paper presented at CHI 2010 Workshop on HCI at the End of Life: Understanding Death, Dying, and the Digital, Atlanta, GA.

Coldwell W. (2013). Why death is not the end of your social media life. The Guardian, 18 February 2013. http://www.theguardian.com/media/shortcuts/2013/feb/18/death-socialmedia-liveson-deadsocial.

Coyne, R. (1998). Cyberspace and Heidegger's pragmatics. Information Technology \& People, 11(4), 338-350.

Dennett, D. (1976). Conditions of Personhood, In Rorty, A.O. (ed) Identities of Persons, Berkeley: University of California Press.

Ebert, R. (2010). TWEET! TWEET! TWEET! Robert Ebert's Journal. 11 June 2010. http://www.rogerebert.com/rogers-journal/tweet-tweet-tweet.

Frow, J. (2012). On Personhood in Public Places. Research Unit in Public Cultures, No. 1, The University of Melbourne. Electronic document available at http://publiccultures.unimelb.edu.au/personhood-public-places

Gayomali, C. (2014) Eterni.me wants to let you Skype your family after you're dead. Fast Company, January, 20, 2014. http://www.fastcompany.com/3025797/tech-forecast/eternimewants-to-let-you-skype-your-family-from-the-grave.

Gell, A. (1998) Art and Agency: An Anthropological Theory. Oxford: Clarendon.

Graham, C., Gibbs, M. \& Aceti, L. (2013) Introduction to the Special Issue on the Death,Afterlife, and Immortality of Bodies and Data, The Information Society: An International Journal, 29(3), 133-141.

Hallam, E, J. Hockey, G. Howarth (1999). Beyond the Body: Death and Social Identity, London: Routledge.

Hallam, E. and Hockey, J. (2001). Death, Memory, and Material Culture. New York: Berg.

Harper, S. (2010) The social agency of dead bodies, Mortality, 15(4), 308-322. 
Hertz, R. (2004 [1907]). Death and the right hand. Abingdon: Routledge.

Hockey, J., C. Komaromy and K. Woodthorpe (2010) The Matter of Death: space, place and materiality, Palgrave.

Jones, C. (2010) Roger Ebert: The Essential Man. Esquire. 16 February, 2010.

http://www.esquire.com/features/roger-ebert-0310.

Kearl M.C. (2010) The proliferation of postselves in American civic and popular cultures, Mortality: Promoting the interdisciplinary study of death and dying, 15(1), 47-63.

Kleenman, J. (2014). Web immortality: the social media sites that keep you alive in the digital world. The Guardian, 7 June, 2014:

http://www.theguardian.com/lifeandstyle/2014/jun/07/web-immortality-social-media-sitesalive-die-digital

Karppi, T. (2013). Noopolitics of memorializing dead facebook users. Culture Machine, 14.

Lambert, A. (2013). Intimacy and friendship on Facebook. Palgrave Macmillan.

Latour, B. (2004). “The Nonhuman.” in S. Harrison, S. Pile and N. Thrift (Eds.) Patterned Ground: Entanglements of Nature and Culture (pp. 224-227). London: Reaktion.

Latour, B. (2005). Reassembling the Social: An Introduction to Actor-Network Theory. Oxford: Oxford University press.

Lifton, R. (1979) The Broken Connection: On Death and the Continuity of Life, Simon \& Schuster.

Light, B. (2014). Disconnecting with Social Networking Sites. Hampshire: Palgrave Macmillan.

Light, B., \& Cassidy, E. (2014). Strategies for the suspension and prevention of connection: Rendering disconnection as socioeconomic lubricant with Facebook. New Media \& Society, doi: 1461444814544002 .

Lombard, M., \& Markaridian Selverian, M. E. (2008). Telepresence after death. Presence: Teleoperators and Virtual Environments, 17(3), 310-325.

Marwick, A. \& Ellison, N. (2012). “There Isn't Wifi in Heaven!” Negotiating Visibility on Facebook Memorial Pages. Journal of Broadcasting \& Electronic Media 56(3), 378-400.

Nansen, B, Arnold, M, Gibbs, M, and Kohn, T. (2014) The restless in the digital cemetery, in C. Moreman and D. Lewis (Eds.) Digital Death: Death: Mortality and Beyond in the Online Age (pp. 111-124), Santa Barbara: Praeger.

Nansen, B, Arnold, M, Gibbs, M, and Kohn, T. (2015, forthcoming) The Stories of Zyzz Distributed Memories on Distributed Networks, In C. Lohmeier, A. Hajek and C. Pentzold (Eds.) Social Media and Social Memories: Remembering in Digitally Networked Times. Palgrave Macmillan.

Odom, W., Harper, R., Sellen, A., Kirk, D., \& Banks, R. (2010). Passing on \& putting to rest: understanding bereavement in the context of interactive technologies. Proceedings of the SIGCHI conference on Human Factors in computing systems, 1831-1840. 
O’Neill, K. (2008) Death, Lives, and Video Streams. Mortality: Promoting the interdisciplinary study of death and dying 13(2): 174-186.

Ostrow, A. (2011). After Your Final Status Update. TED talks, July 2011:

https://www.ted.com/talks/adam_ostrow_after_your_final_status_update?language=en.

Parker, L. (2014) How to become virtually immortal. The New Yorker, 4 April,

2014.http://www.newyorker.com/tech/elements/how-to-become-virtually-immortal.

Russell, N. (2014). Death and Life in Cyberspace. Sunday Star Times, 11 March 2012. http://www.stuff.co.nz/sunday-star-times/latest-edition/6555760/Death-and-life-incyberspace.

Smith, K. (2012) From dividual and individual selves to porous subjects. The Australian Journal of Anthropology, 23(1), 50-64.

Stokes, P. (2012) Ghosts in the Machine: Do the Dead Live on in Facebook? Philosophy \& Technology, 25(3), 363-379.

Tehranian, J. (2011). Parchment, Pixels \& Personhood: User Rights and the IP (Identity Politics) of IP (Intellectual Property). University of Colorado Law Review, 82(1), 1 - 84.

Vigilant, L.G. and John Williamson, J. (2003) Symbolic Immortality and Social Theory: The Relevance of an Underutilized Concept in C.D. Bryant (ed) Handbook of Thanatology: Essays on the Social Study of Death (pp. 173 - 182), Newbury Park, CA: Sage.

Williams, H. (2004). Death Warmed Up: The Agency of Bodies and Bones in Early AngloSaxon Cremation Rites. Journal of Material Culture, Vol. 9(3): 263-291 